1 Secretaria de Estado de Saúde de Minas Gerais (SES-MG) - Belo Horizonte (MG), Brasil. joao_alvespereira@yahoo. com.br

2 Universidade Estadual de Montes Claros (Unimontes) - Montes

Claros (MG), Brasil.

\section{Salário de médicos contratados da Estratégia Saúde da Família e contexto municipal: estudo ecológico}

\author{
Salary of physicians hired by the Family Health Strategy Program and \\ the municipalities' context: an ecological study
}

João Alves Pereira', Renata Fiúza Damasceno', Marta Raquel Mendes Vieira', Alfredo Maurício

Batista de Paula², Desirée Sant'Ana Haikal2

DOI: 10.1590/0103-1104202012603

RESUMO O salário é um importante fator de atração, fixação e satisfação de profissionais médicos na Atenção Primária à Saúde (APS), sobretudo nas regiões mais remotas ou carentes. Este estudo tem o objetivo de identificar características contextuais municipais que estejam associadas ao valor do salário pago a médicos contratados da Estratégia Saúde da Família (ESF). Realizou-se um estudo ecológico e transversal com 78 municípios da Macrorregião de Saúde Norte, Minas Gerais, Brasil. A variável dependente referiu-se ao valor pago aos médicos contratados da ESF. Foram consideradas 22 variáveis independentes contextuais incluindo características sociodemográficas, de localização e indicadores assistenciais e de saúde de cada município, coletados de bases de dados oficiais. Conduziu-se Regressão Linear Múltipla. A média salarial encontrada foi de $\mathrm{R} \$ 11.188,61$ ( $\pm \mathrm{R} \$ 2.093,71)$, variando de $\mathrm{R} \$ 6.388,20$ até $\mathrm{R} \$ 17.000,00$. Após ajuste do modelo, somente a Distância ao Centro Regional manteve-se positivamente associada ao desfecho (R2=6,4\%). O salário dos médicos não foi determinado por características municipais contextuais mensuráveis. Outros fatores mais subjetivos, de difícil mensuração, podem estar envolvidos na questão. Observa-se a necessidade do estabelecimento de parâmetros claros para definição do valor do salário de médicos contratados para atuação na ESF.

PALAVRAS-CHAVE Salários e benefícios. Médicos. Fatores socioeconômicos. Atenção Primária à Saúde. Estratégia Saúde da Família.

\begin{abstract}
Salary is an important factor of attraction, fixation and satisfaction of physicians in Primary Health Care $(\mathrm{PHC})$, mainly in the most remote or poor regions. This study aims to identify characteristics related to municipalities that are associated to salaries paid to physicians hired from the Family Health Strategy (FHS) Program. An ecological and cross-sectional study was conducted with 78 municipalities of the Northern Health Macro-region of Minas Gerais State, Brazil. The dependent variable referred to the amount paid to the physicians hired from FHS. Twenty-two contextual independent variables were applied, including sociodemographic characteristics, location and health and care indicators of each municipality, all collected from public databases. Multiple Linear Regression was conducted. The outcome average salary was $R \$ 11,188.61$ ( $\pm R \$ 2,093.71$ ), ranging from $R \$ 6,388.20$ to $R \$ 17,000.00$. After adjusting the model, only the variable 'Distance from Regional Center' remained positively associated to the outcome ( $R 2=6.4 \%)$. The conclusion is that the salaries of physicians were not determined by measurable contextual municipal characteristics. Other more subjective and difficult to measure factors may be involved in the issue. Therefore, there is need to establish clear parameters so to define the salaries of physicians hired to work for FHS.
\end{abstract}

KEYWORDS Salaries and fringe benefits. Physicians. Socioeconomic factors. Primary Health Care. Family Health Strategy. 


\section{Introdução}

O médico é profissional fundamental para o bom desempenho da Estratégia Saúde da Família (ESF) e, consequentemente, da assistência ofertada à população pelo Sistema Único de Saúde (SUS) $\mathbf{1 , 2}$. A atração e a manutenção desse profissional ainda hoje representam um desafio para consolidação do modelo assistencial defendido para a Atenção Primária à Saúde (APS) ${ }^{3}$. Esse modelo assistencial visa à promoção da equidade no atendimento $\mathrm{e}$ o combate às desigualdades no acesso aos serviços, tendo as práticas orientadas pelos determinantes sociais da saúde e a ESF como eixo estruturante do processo de reorganização do sistema de saúde'.

O salário é um importante fator de atração, fixação e satisfação desses profissionais na $\mathrm{APS}^{4}$, sobretudo nas regiões mais remotas ou carentes $^{3,5}$. Estudos prévios ${ }^{2-6}$ têm investigado fatores que motivam ou atraem os profissionais médicos para atuação na APS tais como localização geográfica, aspectos organizacionais, pessoais, profissionais, condições de trabalho e salário3,6. A pesquisa Demografia Médica no Brasil $2018^{7}$ mostrou que $63,1 \%$ dos médicos pesquisados consideraram o salário como o principal determinante para a fixação do médico em uma instituição ou cidade. Outro estudo de abrangência nacional revelou que 92\% dos médicos consideraram o salário um dos principais fatores para justificar a permanência do profissional em um município ${ }^{3}$.

$\mathrm{O}$ vínculo desses médicos com os serviços de saúde pode se dar por meio de concurso público ou de contratos. Tais contratos ficam a cargo dos gestores municipais de saúde e os tipos mais comuns são os contratos temporários com a administração pública, contratos de prestação de serviços por profissionais autônomos, contratos regidos pelo Regime da Consolidação das Leis do Trabalho (CLT) e demais vínculos sem proteção ou não plenamente protegidos no âmbito do direito do trabalho ${ }^{3}$, comumente conhecidos como contratos precários. Na APS, o salário pago aos médicos com vínculo estável ou permanente (efetivo) é normatizado por leis e geralmente inferior ao de médicos que possuem contratos temporários, considerando a carga horária de 40 horas estabelecida para a ESF${ }^{3}$. O salário dos médicos contratados apresenta uma ampla variação entre municípios, o que possivelmente seria reflexo do contexto social e da necessidade de, diante desses diferentes contextos, atrair e fixar tais profissionais ${ }^{3}$.

Esse cenário aponta oportunidades interessantes para a análise da relação entre fatores contextuais e salários dos médicos que atuam na APS. Em estudos ecológicos, trabalha-se com dados agrupados, coletados em nível coletivo (fatores contextuais), e não em nível individual, sendo a unidade de análise uma área geográfica, como, por exemplo, bairros, escolas ou municípios. Portanto, fatores contextuais referem-se a elementos que compõem o macroambiente dos indivíduos, que afetam seu desempenho, mas não são controláveis por eles ${ }^{8,9}$. Não foram identificados estudos anteriores que tenham investigado a relação entre o salário ofertado aos médicos contratados e sua relação com características municipais, sejam elas relativas a fatores contextuais sociodemográficos, à localização geográfica ou aos indicadores de saúde. Compreender a possível relação entre o salário pago aos médicos contratados da APS e o contexto municipal tem potencial para contribuir para uma maior racionalização e padronização do gasto em saúde. Tal compreensão pode contribuir para a consolidação da ESF, mesmo em contextos menos favoráveis e diante do desafio da alta rotatividade de profissionais na ESF ${ }^{10}$.

Assim, este estudo objetivou identificar possíveis fatores contextuais municipais, sejam eles geográficos, sociodemográficos, assistenciais ou de saúde, que possam exercer influência nos salários pagos aos médicos contratados da ESF.

\section{Material e métodos}

Trata-se de um estudo ecológico e transversal conduzido nos municípios que compõem a 
Macrorregião de Saúde Norte (Macro Norte), uma das treze Macrorregiões de Saúde estabelecidas no Plano Diretor de Regionalização (PDR) da Secretaria de Estado de Saúde de Minas Gerais (SES-MG). A Macro Norte, cujo município-polo é Montes Claros, possui 86 municípios divididos em nove Microrregiões de saúde, totalizando uma população aproximada de 1,6 milhões de habitantes ${ }^{\mathbf{1 1}}$. Trata-se de uma região caracterizada por possuir baixos indicadores sociais, marcante desigualdade social, grande extensão territorial, população predominantemente rural, baixa densidade demográfica e significativas distâncias entre municípios ${ }^{\mathbf{1 2}, 13}$. Possui o município com o menor Índice de Desenvolvimento Humano Municipal (IDH-M) do Estado ${ }^{\mathbf{1 4}}$.

Montes Claros é um município de médio porte, que possui uma população de 390.212 habitantes (2014) e elevado IDH-M (2010). É considerado polo regional por ser referência na assistência ambulatorial e hospitalar para os 86 municípios da Macro Norte ${ }^{15}$. O município se destaca por abrigar instituições e oferecer serviços e atividades diversificadas que não são encontrados em outros municípios da região, constituindo-se um importante polo universitário do estado ${ }^{16}$.

Os dados foram coletados em duas etapas. A primeira etapa, realizada nos meses de novembro e dezembro de 2017, refere-se à coleta de dados contextuais dos municípios da Macro Norte, extraídos de banco de dados oficiais de acesso público (variáveis independentes).
A segunda etapa, realizada nos meses de janeiro e fevereiro de 2018, refere-se à coleta de informações sobre o salário dos médicos contratados pela ESF dos municípios pertencentes à Macro Norte (variável dependente). Tais informações foram obtidas de banco de dados da Superintendência Regional de Saúde de Montes Claros (SRS-Montes Claros), uma unidade regional da SES-MG. Considerou-se, para fins deste estudo, o valor do maior salário pago aos médicos contratados que atuavam nas equipes da ESF em cada município, por apresentar maior variação nos valores quando comparados aos salários dos médicos efetivos.

A variável Número de Médicos por Mil Habitantes foi calculada a partir da metodologia preconizada pela Rede Interagencial de Informações para o Brasil (Ripsa) para os Indicadores e Dados Básicos para a Saúde no Brasil (IDB) ${ }^{\mathbf{1 7}}$. Os dados referentes ao número de médicos foram coletados por meio da ferramenta Tabnet, acessível no site do Departamento de Informática do SUS (Datasus), e referem-se ao mês de outubro de 201718 . Os dados sobre população foram coletados no site do Instituto Brasileiro de Geografia e Estatística (IBGE) e referem-se ao ano de 2017 , com ajuste para $1^{\circ}$ de julho ${ }^{19}$.

Os dados coletados sobre contexto sociodemográfico, localização geográfica e indicadores assistenciais e de saúde dos municípios da Macro Norte (variáveis independentes) foram agrupados em blocos temáticos (quadro 1). 
Quadro 1. Variáveis independentes por blocos temáticos considerando a localização geográfica, o contexto sociodemográfico e os indicadores assistenciais e de saúde dos municípios da Macrorregião de Saúde Norte, Minas Gerais, Brasil

\begin{tabular}{|c|c|c|c|c|}
\hline $\begin{array}{l}\text { Bloco temá- } \\
\text { tico }\end{array}$ & Variável & $\begin{array}{l}\text { Ano de } \\
\text { referência }\end{array}$ & Fonte & Conceito \\
\hline $\begin{array}{l}\text { Localização } \\
\text { geográfica }\end{array}$ & $\begin{array}{l}\text { Distância ao Centro } \\
\text { Regional }\end{array}$ & 2017 & $\begin{array}{l}\text { Google Maps https://www. } \\
\text { google.com.br/maps/ }\end{array}$ & $\begin{array}{l}\text { Distância em Km relativa ao trajeto mais rápido entre o muni- } \\
\text { cípio estudado e Montes Claros (município-polo da região). }\end{array}$ \\
\hline \multirow{8}{*}{$\begin{array}{l}\text { Contexto } \\
\text { sociodemo- } \\
\text { gráfico }\end{array}$} & População & 2010 & $\begin{array}{l}\text { IBGE (Censo 2010) https:// } \\
\text { cidades.ibge.gov.br/ }\end{array}$ & Número de habitantes do município. \\
\hline & $\begin{array}{l}\text { Densidade demo- } \\
\text { gráfica }\end{array}$ & 2010 & $\begin{array}{l}\text { IBGE (Censo 2010) https:// } \\
\text { cidades.ibge.gov.br/ }\end{array}$ & $\begin{array}{l}\text { Relação entre o número de habitantes de um determinado } \\
\text { local com a sua área em } \mathrm{Km}^{2} \text {. }\end{array}$ \\
\hline & IDH-M & 2010 & $\begin{array}{l}\text { IBGE (Censo 2010) https:// } \\
\text { cidades.ibge.gov.br/ }\end{array}$ & $\begin{array}{l}\text { O Índice de Desenvolvimento Humano Municipal (IDH-M) } \\
\text { é uma medida composta de indicadores de três dimensões: } \\
\text { longevidade, educação e renda. Varia de } 0 \text { a } 1 \text { e quanto mais } \\
\text { próximo de } 1 \text {, maior o desenvolvimento humano. }\end{array}$ \\
\hline & Índice de Gini & 2010 & $\begin{array}{l}\text { IBGE (Censo 2010) https:// } \\
\text { cidades.ibge.gov.br/ }\end{array}$ & $\begin{array}{l}\text { Medida do grau de concentração de uma distribuição, cujo } \\
\text { valor varia de zero (perfeita igualdade) até um (desigualdade } \\
\text { máxima). }\end{array}$ \\
\hline & $\begin{array}{l}\text { População em extre- } \\
\text { ma pobreza }\end{array}$ & $\begin{array}{l}2010 \text { (Agos- } \\
\text { to) }\end{array}$ & $\begin{array}{l}\text { Atlas do Desenvolvimento } \\
\text { Humano no Brasil http:// } \\
\text { atlasbrasil.org.br/2013/pt/ }\end{array}$ & $\begin{array}{l}\text { Proporção (\%) de indivíduos com renda domiciliar per capita } \\
\text { mensal igual ou inferior a } R \$ 70,00 \text {. O universo é limitado aos } \\
\text { indivíduos de domicílios particulares permanentes. }\end{array}$ \\
\hline & População rural & 2010 & $\begin{array}{l}\text { Atlas do Desenvolvimento } \\
\text { Humano no Brasil http:// } \\
\text { atlasbrasil.org.br/2013/pt/ }\end{array}$ & $\begin{array}{l}\text { Porcentagem da população do município que vive em áreas } \\
\text { rurais. }\end{array}$ \\
\hline & IMRS & 2010 & $\begin{array}{l}\text { Fundação João Pinheiro } \\
\text { http://fjp.mg.gov.br/ }\end{array}$ & $\begin{array}{l}\text { O Índice Mineiro de Responsabilidade Social (IMRS) é o } \\
\text { resultado da média ponderada dos índices de dez dimensões, } \\
\text { incluindo saúde, educação, emprego e renda, que agregam } \\
\text { indicadores municipais dos três anos anteriores ao do cálculo. }\end{array}$ \\
\hline & IVS & 2010 & $\begin{array}{l}\text { Ipea http://ivs.ipea.gov.br/ } \\
\text { index.php/pt/ }\end{array}$ & $\begin{array}{l}\text { O Índice de Vulnerabilidade Social (IVS) é o resultado da } \\
\text { média aritmética dos subíndices: IVS Infraestrutura Urbana, } \\
\text { IVS Capital Humano e IVS Renda e Trabalho, calculados a } \\
\text { partir de } 16 \text { indicadores. }\end{array}$ \\
\hline \multirow{7}{*}{$\begin{array}{l}\text { Indicadores } \\
\text { assisten- } \\
\text { ciais e de } \\
\text { saúde }\end{array}$} & $\begin{array}{l}\text { População com plano } \\
\text { de saúde }\end{array}$ & 2017 (Junho) & $\begin{array}{l}\text { DAB/MS http://dab2.saude. } \\
\text { gov.br/sistemas/notatecnica/ } \\
\text { frmListaMunic.php }\end{array}$ & $\begin{array}{l}\text { Razão, expressa em porcentagem, do número de beneficiários } \\
\text { em relação à população em uma área específica. }\end{array}$ \\
\hline & $\begin{array}{l}\text { eAB muito acima da } \\
\text { média no PMAQ-AB }\end{array}$ & 2017 & $\begin{array}{l}\text { DAB/MS http://dab2.saude. } \\
\text { gov.br/sistemas/notatecnica/ } \\
\text { frmListaMunic.php }\end{array}$ & $\begin{array}{l}\text { Porcentagem das equipes de Atenção Básica (eAB) que parti- } \\
\text { ciparam do } 2^{\circ} \text { ciclo do PMAQ-AB certificadas com desempe- } \\
\text { nho muito acima da média. }\end{array}$ \\
\hline & $\begin{array}{l}\text { Número de médicos } \\
\text { por mil habitantes }\end{array}$ & 2017 & $\begin{array}{l}\text { Calculado pelos autores } \\
\text { Ripsa/Datasus/IBGE }\end{array}$ & $\begin{array}{l}\text { Número de médicos em atividade, por mil habitantes, em } \\
\text { determinado espeço geográfico, no ano considerado. }\end{array}$ \\
\hline & $\begin{array}{l}\text { Despesa total com } \\
\text { saúde por habitante }\end{array}$ & 2016 & $\begin{array}{l}\text { Siops http://portalsaude. } \\
\text { saude.gov.br/index.php/o- } \\
\text {-ministerio/principal/siops }\end{array}$ & $\begin{array}{l}\text { Despesa liquidada total com saúde per capita declarada pelos } \\
\text { municípios ao Ministério da Saúde por meio do Sistema de } \\
\text { Informações sobre Orçamentos Públicos em Saúde (Siops). }\end{array}$ \\
\hline & Presença de hospital & 2017 & $\begin{array}{l}\text { CNES http://cnes.datasus. } \\
\text { gov.br/ }\end{array}$ & $\begin{array}{l}\text { Municípios que possuem hospital municipal ou microrregio- } \\
\text { nal funcionando e com cadastrado ativo no Cadastro Nacio- } \\
\text { nal de Estabelecimentos de Saúde (CNES). }\end{array}$ \\
\hline & $\begin{array}{l}\text { Cobertura populacio- } \\
\text { nal estimada de ESF }\end{array}$ & $\begin{array}{l}2017 \text { (Outu- } \\
\text { bro) }\end{array}$ & $\begin{array}{l}\text { DAB/MS http://dab2.saude. } \\
\text { gov.br/sistemas/notatecnica/ } \\
\text { frmListaMunic.php }\end{array}$ & $\begin{array}{l}\text { Estimativa da cobertura populacional por equipes da estra- } \\
\text { tégia saúde da família, que considera a população de } 3000 \\
\text { habitantes por equipe. }\end{array}$ \\
\hline & $\begin{array}{l}\text { Proporção de inter- } \\
\text { nações por condições } \\
\text { sensíveis a APS }\end{array}$ & 2015 & $\begin{array}{l}\text { Tabnet/Datasus http:// } \\
\text { tabnet.datasus.gov.br/cgi/ } \\
\text { tabcgi.exe?pacto/2015/cnv/ } \\
\text { coapmunmg.def }\end{array}$ & $\begin{array}{l}\text { Proporção entre o no de internações por causas selecionadas } \\
\text { sensíveis à atenção básica, por local de residência e ano de } \\
\text { internação, e total de internações clínicas, por local de resi- } \\
\text { dência e ano de internação. }\end{array}$ \\
\hline
\end{tabular}


Quadro 1. (cont.)

\begin{tabular}{|c|c|c|c|c|}
\hline $\begin{array}{l}\text { Bloco temá- } \\
\text { tico }\end{array}$ & Variável & $\begin{array}{l}\text { Ano de } \\
\text { referência }\end{array}$ & Fonte & Conceito \\
\hline \multirow[t]{6}{*}{$\begin{array}{l}\text { Indicadores } \\
\text { assisten- } \\
\text { ciais e de } \\
\text { saúde }\end{array}$} & $\begin{array}{l}\text { Proporção de nasci- } \\
\text { dos vivos de mães } \\
\text { com } 7 \text { ou mais con- } \\
\text { sultas de pré-natal }\end{array}$ & 2014 & $\begin{array}{l}\text { Tabnet/Datasus http:// } \\
\text { tabnet.datasus.gov.br/cgi/ } \\
\text { tabcgi.exe?pacto/2015/cnv/ } \\
\text { coapmunmg.def }\end{array}$ & $\begin{array}{l}\text { Proporção entre o no de nascidos vivos de mães residentes } \\
\text { em determinado local e ano com sete ou mais consultas de } \\
\text { pré-natal e o no de nascidos vivos de mães residentes no } \\
\text { mesmo local e período. }\end{array}$ \\
\hline & $\begin{array}{l}\text { Coberturas vacinais } \\
\text { alcançadas }\end{array}$ & 2014 & $\begin{array}{l}\text { Tabnet/Datasus http:// } \\
\text { tabnet.datasus.gov.br/cgi/ } \\
\text { tabcgi.exe?pacto/2015/cnv/ } \\
\text { coapmunmg.def }\end{array}$ & $\begin{array}{l}\text { Proporção entre o número de vacinas do Calendário Básico } \\
\text { de Vacinação da Criança com coberturas vacinais alcançadas } \\
\text { e o no - total de vacinas constantes no mesmo calendário em } \\
\text { determinado local e ano. }\end{array}$ \\
\hline & $\begin{array}{l}\text { Citopatológico do } \\
\text { colo uterino }\end{array}$ & 2015 & $\begin{array}{l}\text { Tabnet/Datasus http:// } \\
\text { tabnet.datasus.gov.br/cgi/ } \\
\text { tabcgi.exe?pacto/2015/cnv/ } \\
\text { coapmunmg.def }\end{array}$ & $\begin{array}{l}\text { Razão de exames citopatológicos do colo do útero em mulhe- } \\
\text { res de } 25 \text { a } 64 \text { anos pela população da mesma faixa etária em } \\
\text { determinado local e ano. }\end{array}$ \\
\hline & $\begin{array}{l}\text { Mamografia de ras- } \\
\text { treamento }\end{array}$ & 2015 & $\begin{array}{l}\text { Tabnet/Datasus http:// } \\
\text { tabnet.datasus.gov.br/cgi/ } \\
\text { tabcgi.exe?pacto/2015/cnv/ } \\
\text { coapmunmg.def }\end{array}$ & $\begin{array}{l}\text { Razão de exames de mamografia de rastreamento realizados } \\
\text { em mulheres de } 50 \text { a } 69 \text { anos pela população da mesma } \\
\text { faixa etária em determinado local e ano. }\end{array}$ \\
\hline & $\begin{array}{l}\text { Cobertura de acom- } \\
\text { panhamento do Pro- } \\
\text { grama Bolsa Família }\end{array}$ & 2015 & $\begin{array}{l}\text { Tabnet/Datasus http:// } \\
\text { tabnet.datasus.gov.br/cgi/ } \\
\text { tabcgi.exe?pacto/2015/cnv/ } \\
\text { coapmunmg.def }\end{array}$ & $\begin{array}{l}\text { Cobertura de acompanhamento das condicionalidades de } \\
\text { saúde do Programa Bolsa Família (PBF), que indica o no de } \\
\text { famílias beneficiárias com perfil saúde acompanhadas pela } \\
\text { atenção básica na última vigência do ano. }\end{array}$ \\
\hline & $\begin{array}{l}\text { Proporção de parto } \\
\text { normal }\end{array}$ & 2014 & $\begin{array}{l}\text { Tabnet/Datasus http:// } \\
\text { tabnet.datasus.gov.br/cgi/ } \\
\text { tabcgi.exe?pacto/2015/cnv/ } \\
\text { coapmunmg.def }\end{array}$ & $\begin{array}{l}\text { Proporção entre o no de nascidos vivos por parto normal } \\
\text { ocorridos, de mães residentes em determinado local e ano e } \\
\text { o no de nascidos vivos de todos os partos, de mães residentes } \\
\text { no mesmo local e ano. }\end{array}$ \\
\hline
\end{tabular}

IBGE - Instituto Brasileiro de Geografia e Estatística.

Ipea - Instituto de Pesquisa Econômica Aplicada.

DAB/MS - Departamento de Atenção Básica do Ministério da Saúde.

PMAQ-AB - Programa de Melhoria do Acesso e da Qualidade da Atenção Básica.

Para análise dos dados utilizou-se o programa estatístico Statistical Package for the Social Sciences (SPSS) ${ }^{\circledR}$, Versão 18. Foram excluídos das análises oito municípios que não informaram o valor do salário pago aos médicos contratados ou que não possuíam médicos nessa condição; assim, as análises foram conduzidas considerando 78 dos 86 municípios da Macro Norte. Inicialmente, conduziu-se análise exploratória da variável dependente considerando toda a Macro Norte e por região de saúde que a compõe. Foi também conduzida análise descritiva de todas as variáveis independentes, com apresentação de medidas de tendência central e de dispersão para as variáveis numéricas e frequência simples e relativa para as variáveis categóricas. Foi conduzido teste de multicolinearidade entre as variáveis independentes a fim de testar a possível existência de alta correlação $(r \geq 0,70)$ entre elas. Na sequência, foi conduzida análise bivariada por meio da correlação de Pearson (para variável independente numérica) e Teste T ou Anova (para as variáveis independentes categóricas). As variáveis independentes que se mostraram associadas à variável dependente ao nível de significância de $20 \%(\alpha=0,2)$ foram selecionadas para compor o modelo múltiplo. Para tanto, foram realizadas análises por meio da Regressão Linear. O modelo foi ajustado, 
mantendo-se somente as variáveis que se mostraram associadas ao desfecho em nível de significância de $5 \%(\alpha=0,05)$.

Ao final, testaram-se os pressupostos para a regressão linear: linearidade dos parâmetros (diagrama de dispersão e coeficiente de correlação), homocedasticidade (análise gráfica de resíduos), independência dos termos (Teste de Durbin-Watson), normalidade na distribuição dos resíduos (gráfico de probabilidade normal para os resíduos, Testes de KolmogorovSmirnov e Shapiro-Wilk) e ausência de multicolinearidade (análise do valor da tolerância e Fator de Inflação de Variância) ${ }^{\mathbf{2 0}}$.

O estudo seguiu as diretrizes e normas da Resolução no 466/2012 do Conselho Nacional de Saúde, que regulamenta os aspectos éticos e legais das pesquisas no Brasil, sendo apreciado e aprovado pelo Comitê de Ética em Pesquisa da Universidade Estadual de Montes Claros (Unimontes), sob o Parecer nº 2.112.319.

\section{Resultados}

A média do salário bruto pago aos médicos contratados na Macro Norte foi de R $\$ 11.188,61$, sendo que 35 (45\%) municípios pagavam salário abaixo dessa média.

A figura 1 apresenta a distribuição espacial das médias salariais nas nove regiões de saúde que compõem a Macro Norte. A maior média salarial foi verificada na região de saúde Manga, enquanto a menor foi observada na região de saúde Brasília de Minas-São Francisco (tabela 1). A maior variação salarial em uma mesma região de saúde foi observada na região de saúde Montes Claros-Bocaiúva (tabela 1). Os municípios que apresentaram o maior e o menor salário estavam localizados, respectivamente, nas regiões de saúde Salinas-Taiobeiras e Montes Claros-Bocaiúva (tabela 1).

Figura 1. Média salarial por região de saúde da Macrorregião de Saúde Norte, Minas Gerais, Brasil, 2019

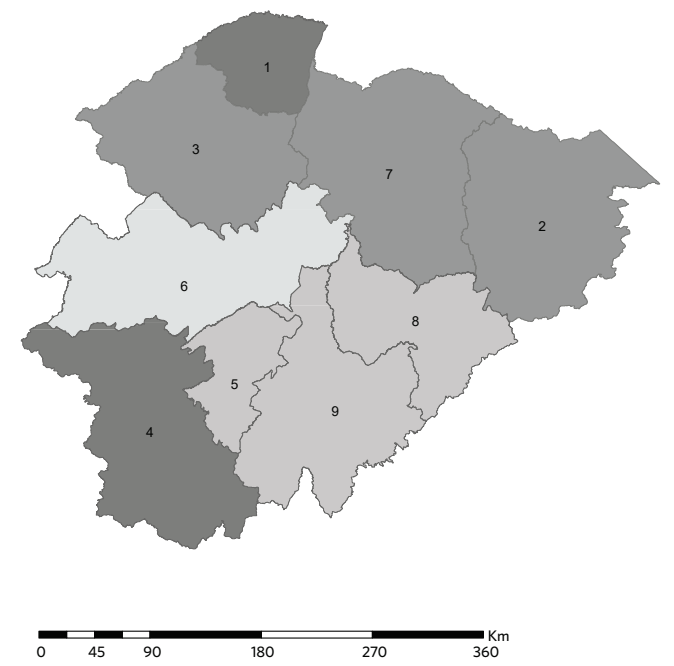

1. Manga

2. Salinas/Taiobeiras

3. Januária

4. Pirapora

5. Coração de Jesus

6. Brasília de Minas/São Francisco

7. Janaúba/Monte Azul

8. Francisco Sá

9. Montes Claros/Bocaiúva

Média Salarial

9973,91

9973,92 - 10936,00

10936,01 - 11889,39

$11889,40-13150,60$

Fonte: Elaboração própria. 
A tabela 1 apresenta também a análise descritiva das variáveis independentes. Observou-se que, na Macro Norte, a distância média dos municípios a Montes Claros foi de aproximadamente $186 \mathrm{Km}$; a população média dos municípios foi de aproximadamente 19 mil habitantes; 97,4\% dos municípios apresentaram IDH-M baixo ou médio; em média, $19 \%$ da população dos municípios vivia em extrema pobreza; $45 \%$ da população dos municípios vivia em áreas rurais; e apenas $2 \%$ da população dos municípios possuía plano de saúde. Apenas 26 municípios (37,1\%) possuíam hospital.

Tabela 1. Análises descritivas da variável dependente e das variáveis independentes relativas à localização geográfica, ao contexto sociodemográfico e aos indicadores assistenciais e de saúde dos municípios $(n=78)$ da Macrorregião de Saúde Norte, Minas Gerais, Brasil

\begin{tabular}{|c|c|c|c|c|c|c|c|c|c|}
\hline Variável & $\mathbf{n}$ & Média & DP & CV(\%) & Mínimo & $\mathbf{P}_{25}$ & Mediana & $\mathbf{P}_{75}$ & Máximo \\
\hline \multicolumn{10}{|l|}{ Variável dependente } \\
\hline Salário bruto do médico contratado & 78 & $11.188,61$ & $2.093,71$ & 18,7 & $6.388,20$ & $9.900,00$ & $11.000,00$ & $12.245,00$ & $17.000,00$ \\
\hline \multicolumn{10}{|l|}{ Região de Saúde } \\
\hline Brasília de Minas/São Francisco & 14 & $9.973,91$ & $1.639,21$ & 16,4 & $8.000,00$ & $8.000,00$ & $9.950,00$ & $10.999,89$ & $13.215,03$ \\
\hline Coração de Jesus & 5 & $10.936,00$ & $1.456,18$ & 13,3 & $9.500,00$ & $9.750,00$ & $10.500,00$ & $12.340,00$ & $13.180,00$ \\
\hline Francisco Sá & 6 & $10.716,66$ & $2.628,43$ & 24,5 & $7.500,00$ & $8.962,50$ & $9.750,00$ & $13.887,50$ & $14.000,00$ \\
\hline Janaúba/Monte Azul & 15 & $11.264,56$ & $1.768,33$ & 15,7 & $8.172,90$ & $10.000,00$ & $11.000,00$ & $12.000,00$ & $14.206,00$ \\
\hline Januária & 4 & $11.251,00$ & $2.552,31$ & 22,7 & $8.240,00$ & $8.796,00$ & $11.232,00$ & $13.725,00$ & $14.300,00$ \\
\hline Manga & 4 & $13.150,60$ & $2.458,76$ & 18,7 & $10.064,00$ & $10.745,10$ & $13.269,20$ & $15.437,50$ & $16.000,00$ \\
\hline Montes Claros/Bocaiúva & 10 & $10.660,75$ & $2.742,91$ & 25,7 & $6.388,20$ & $9.101,51$ & $10.536,67$ & $11.785,00$ & $16.600,00$ \\
\hline Pirapora & 5 & $12.514,95$ & $1.424,70$ & 11,4 & $10.970,30$ & $11.385,15$ & $12.000,00$ & $13.902,22$ & $14.646,48$ \\
\hline Salinas/Taiobeiras & 15 & $11.889,33$ & $1.803,45$ & 15,2 & $9.000,00$ & $11.000,00$ & $12.000,00$ & $12.500,00$ & $17.000,00$ \\
\hline \multicolumn{10}{|l|}{ Variáveis independentes } \\
\hline Distância ao Centro Regional (Km) & 78 & 185,79 & 85,49 & 46,0 & 0,0 & 110,50 & 175,50 & 251,50 & 374,00 \\
\hline População (Hab) & 78 & $18.977,61$ & $41.731,96$ & 219,9 & $3.684,00$ & $5.675,75$ & $7.922,00$ & $20.358,25$ & $361.915,00$ \\
\hline Densidade Demográfica (Hab/Km2) & 78 & 14,23 & 14,42 & 101,4 & 2,00 & 7,00 & 10,00 & 17,25 & 111,00 \\
\hline IDH-M & 78 & 0,624 & 0,038 & 06,1 & 0,529 & 0,604 & 0,624 & 0,643 & 0,770 \\
\hline Índice de Gini & 78 & 0,491 & 0,053 & 10,8 & 0,400 & 0,450 & 0,480 & 0,520 & 0,680 \\
\hline População em extrema pobreza (\%) & 78 & 19,19 & 8,48 & 44,2 & 3,40 & 13,19 & 18,24 & 23,30 & 42,79 \\
\hline População rural (\%) & 78 & 45,19 & 18,11 & 40,1 & 4,83 & 33,22 & 45,29 & 58,75 & 79,83 \\
\hline IMRS & 78 & 0,533 & 0,029 & 05,5 & 0,462 & 0,510 & 0,536 & 0,551 & 0,610 \\
\hline IVS & 78 & 0,431 & 0,071 & 16,5 & 0,220 & 0,385 & 0,432 & 0,477 & 0,631 \\
\hline População com plano de saúde (\%) & 78 & 2,20 & 3,40 & 154,3 & 0,05 & 0,36 & 0,98 & 1,94 & 21,48 \\
\hline eAB muito acima da média no PMAQ-AB (\%) & 78 & 17,92 & 27,40 & 152,9 & 0,0 & 0,0 & 0,0 & 34,60 & 100,00 \\
\hline Número de médicos por mil habitantes & 78 & 0,59 & 0,39 & 66,1 & 0,10 & 0,39 & 0,49 & 0,73 & 2,98 \\
\hline Despesa total com saúde por habitante & 78 & 586,25 & 137,06 & 23,4 & 367,12 & 510,38 & 567,80 & 638,02 & $1.123,38$ \\
\hline Cobertura populacional estimada de ESF & 78 & 99,64 & 1,617 & 01,6 & 89,90 & 100,00 & 100,00 & 100,00 & 100,00 \\
\hline Internações por condições sensíveis a APS (\%) & 78 & 28,53 & 8,833 & 31,0 & 14,75 & 22,01 & 26,39 & 33,48 & 55,66 \\
\hline Nascidos vivos com sete ou mais consultas (\%) & 78 & 78,13 & 10,697 & 13,7 & 45,00 & 71,95 & 79,33 & 86,10 & 96,00 \\
\hline Coberturas vacinais alcançadas & 78 & 71,22 & 22,326 & 31,3 & 0,0 & 66,67 & 77,78 & 88,89 & 100,00 \\
\hline Citopatológico do colo uterino & 78 & 0,73 & 0,278 & 38,1 & 0,07 & 0,54 & 0,70 & 0,89 & 1,49 \\
\hline Mamografia de rastreamento & 78 & 0,62 & 0,507 & 81,8 & 0,01 & 0,20 & 0,39 & 1,06 & 1,82 \\
\hline Cobertura de acompanhamento do PBF & 78 & 85,05 & 11,244 & 13,2 & 41,70 & 82,04 & 88,11 & 92,23 & 100,00 \\
\hline Proporção de parto normal & 78 & 58,45 & 10,02 & 17,1 & 36,17 & 52,60 & 58,21 & 66,69 & 79,40 \\
\hline
\end{tabular}

$\mathrm{n}=$ Número; $\mathrm{DP}=$ Desvio Padrão; $C V=$ Coeficiente de Variação; $P_{25}=$ Percentil 25; $P_{75}=$ Percentil 75. 
A tabela 2 apresenta as análises bivariadas. Para compor o modelo múltiplo do grupo de variáveis relativas ao contexto sociodemográfico, foram selecionadas, ao nível de significância de $20 \%$, as variáveis IDH-M e IVS; do grupo relativo à localização, foi selecionada a variável Distância ao Centro Regional; e do grupo de variáveis relativas aos indicadores assistenciais e de saúde, foram selecionadas as variáveis Citopatológico do Colo Uterino e Proporção de Parto Normal. As variáveis Distância ao Centro
Regional, IVS e Citopatológico do Colo uterino apresentaram correlação positiva, enquanto as variáveis IDH-M e Proporção de Parto Normal apresentaram correlação negativa (tabela 2). No teste de multicolinearidade, observou-se elevada correlação somente entre as variáveis independentes População e Densidade Demográfica $(\mathrm{r}=0,788)$, mas que não apresentaram associação com o salário dos médicos contratados; não foram, portanto, selecionadas para a análise múltipla.

Tabela 2. Análise de correlação entre o salário bruto do profissional médico contratado e as variáveis relativas à localização geográfica, ao contexto sociodemográfico e aos indicadores assistenciais e de saúde, Macrorregião de Saúde Norte, Minas Gerais, Brasil

\begin{tabular}{lrr}
\hline Indicadores & $\begin{array}{r}\text { Coeficiente de Cor- } \\
\text { relação de Pearson }\end{array}$ & $\begin{array}{r}\text { Nível de significância } \\
\text { (p valor) }\end{array}$ \\
\hline Distância ao Centro Regional (Km) & 0,276 & 0,014 \\
População (Hab) & $-0,021$ & 0,855 \\
Densidade Demográfica (Hab/Km2) & 0,464 \\
IDH-M & $-0,084$ & 0,067 \\
Índice de Gini & $-0,209$ & 0,511 \\
População em extrema pobreza (\%) & $-0,076$ & 0,274 \\
População rural (\%) & 0,125 & 0,697 \\
IMRS & $-0,045$ & 0,496 \\
IVS & $-0,078$ & 0,050 \\
População com plano de saúde (\%) & 0,223 & 0,528 \\
eAB muito acima da média no PMAQ-AB (\%) & 0,441 \\
Número de médicos por mil habitantes & $-0,073$ & 0,264 \\
Despesa total com saúde por habitante & 0,089 & 0,944 \\
Cobertura populacional estimada de ESF & 0,128 & 0,511 \\
Internações por condições sensíveis a APS (\%) & 0,008 & 0,253 \\
Nascidos vivos com sete ou mais consultas (\%) & 0,076 & 0,522 \\
Cobertura vacinal alcançada & 0,131 & 0,709 \\
Citopatológico do colo uterino & 0,074 & 0,166 \\
Mamografia de rastreamento & 0,0469 \\
Condicionalidades do PBF & 0,158 & 0,851 \\
Proporção de parto normal & $-0,034$ & 0,022 \\
\hline
\end{tabular}

Na tabela 3, observa-se o modelo de regressão múltipla ajustado. A variável Distância ao Centro Regional $(\mathrm{p}=0,014)$ foi a única que permaneceu no modelo final (r2=0,064).
As estimativas apontaram que, em média, o aumento de cada Km de distância ao centro regional acarreta acréscimo de $\mathrm{R} \$ 6,75$ no salário. 
Tabela 3. Modelo de regressão linear múltipla relativo ao salário bruto do profissional médico contratado dos municípios da Macrorregião de Saúde Norte, Minas Gerais, Brasil

\begin{tabular}{lrrrrr}
\hline Modelo por ordem de entrada das & \multicolumn{4}{c}{ Coeficientes } \\
\cline { 2 - 5 } variáveis & Estimativa $(\boldsymbol{\beta})$ & Erro padrão & p-valor & \multicolumn{1}{c}{ IC 95\% } \\
\hline Constante & $9.932,986$ & 551,598 & 0,000 & $8.834,381$ & $11.031,586$ \\
Distância ao Centro Regional $(\mathrm{Km})$ & 6,758 & 2,700 & 0,014 & 1,38 & 12,14 \\
\hline
\end{tabular}

$R^{2}$ ajustado $=0,064$

Nos testes dos pressupostos para a regressão linear, verificaram-se a linearidade dos parâmetros $(r<0,3)$, a independência dos termos ( $D W=2,1 ; p \leq 0,05)$ e a ausência de multicolinearidade (tolerâncias $>0,10$ e VIF $<10$ ). Observaram-se também homocedasticidade e normalidade na distribuição dos resíduos (KS=0,091; $\mathrm{p}=0,173)$.

\section{Discussão}

Este estudo revelou que o único fator contextual municipal a exercer influência sobre os valores dos salários pagos aos médicos contratados foi a Distância ao Centro Regional, município-polo da Macro Norte, apesar de diversas variáveis contextuais relativas à localização geográfica, ao contexto sociodemográfico e aos indicadores assistenciais e de saúde terem sido testadas. O modelo final apresentou capacidade de predição do desfecho de somente $6,4 \%$, sugerindo que outros fatores, diferentes dos considerados neste estudo, estejam envolvidos na determinação dos salários pagos aos médicos contratados da ESF.

Em estudos ecológicos, que investigam a influência do contexto em questões específicas, é frequente a identificação de indicadores de desigualdade de renda ${ }^{21-23}$, de desenvolvimento humano ${ }^{22-24} \mathrm{e}$ assistenciais ${ }^{25-27}$ influenciando questões relacionadas à saúde $22,23,25 \mathrm{e}$ asssistência23,27,28. Dessa forma, acreditava-se, inicialmente, que os salários pagos aos médicos contratados também seriam influenciados por tais indicadores, o que, na prática, não se confirmou. Possivelmente, questões subjetivas tais como vontade política ${ }^{29}$ ou de ordem pessoal, não passíveis de serem captadas ou mensuradas por indicadores municipais convencionais comumente utilizados para retratar as realidades, sejam os reais determinantes dos salários pagos aos médicos contratados.

A correlação positiva entre o salário bruto do médico contratado e a variável independente Distância ao Centro Regional indica uma tendência dos municípios que estão localizados mais distantes geograficamente dos centros regionais e que comumente apresentam escassez de profissionais, de ofertarem maiores salários como forma de atrair e fixar médicos para a ESF. A maior média salarial foi observada na região de saúde Manga, justamente a região mais distante do centro regional, que é Montes Claros, e que apresenta maiores barreiras geográficas, incluindo a travessia do rio São Francisco por meio de balsa. Esse achado encontra-se em conformidade com outros estudos 3 ,6,30,31, que afirmam que o salário exerce forte impacto sobre a preferência do emprego e que o aumento do salário é um fator de atração de médicos na APS. Como há uma preferência por cidades de médio e grande porte, a oferta de um salário diferenciado é fator importante para atrair médicos para a APS em áreas remotas e/ou desassistidas do interior ${ }^{30}$.

Ainda que o fator Distância ao Centro Regional explique apenas $6,4 \%$ da variabilidade do salário pago aos profissionais médicos contratados da ESF, reforça os achados de outros estudos ${ }^{6,10,32}$, que demostraram que as 
melhores propostas salariais são oriundas de regiões longínquas e pouco atrativas socioeconomicamente. Entretanto, convém ressaltar que os altos salários conseguem atrair, mas não fixar os médicos, que migram em busca de melhores condições de trabalho e de satisfação pessoal e profissional6,10,32. A oferta de altos salários pelos municípios tem estimulado a competição entre os mesmos e, consequentemente, fomentado a alta rotatividade de médicos da APS 3,6,32.

A média salarial na Macro Norte foi de aproximadamente $\mathrm{R} \$ 11.000,00$, sendo observada menor variabilidade de médias salariais entre as regiões de saúde ( $\mathrm{CV}=8,7 \%$, dados não mostrados) do que entre os municípios de uma mesma região de saúde ( $\mathrm{CV}=18,7 \%)$. A média salarial dos médicos contratados verificada neste estudo se aproximou do valor da bolsa paga aos médicos do PMMB, que, na época do levantamento dos dados (jan-fev de 2018), era de $R \$ 11.865,60$, o que poderia sugerir que esse valor fosse, de alguma forma, utilizado como parâmetro auxiliar na definição dos salários pagos. No entanto, há que se considerar que a amplitude de variação dos salários ultrapassou $\mathrm{R} \$ 10.000,00$, o que denota alta variação. Embora os municípios tenham apresentado contexto sociodemográfico semelhante, os indicadores geográficos e assistenciais e de saúde revelaram grande variação, observada pelo CV. Entretanto, tais indicadores contextuais não foram suficientes para explicar a variabilidade do valor do salário pago aos médicos.

O médico é profissional fundamental para a consolidação da ESF como forma de reorganização da APS no Brasil, e sua atuação contribui decisivamente para a resolutividade da APS. A atração e manutenção desses profissionais para a ESF representam hoje um dos grandes desafios estruturantes do SUS $2,5,6$. Na forma de contratação ou ingresso desse profissional na ESF reside um importante nó crítico a ser considerado, já que há evidências de que a vinculação por meio de concurso público aumenta o tempo de permanência desses profissionais na APS6. Também existe uma discrepância entre os valores pagos aos médicos efetivos e contratados, com uma média salarial maior entre estes últimos ${ }^{3}$.

O salário do médico exerce alto impacto nos custos de uma equipe da ESF, já que, na prática, o valor pago a esse profissional geralmente já é superior ao valor mensal transferido pelo Ministério da Saúde (MS) ao município para contribuir com o custeio de uma equipe de saúde da família. É importante ressaltar que o custeio de uma equipe da ESF abrange, além do pagamento de todos os profissionais da equipe, as despesas relacionadas ao funcionamento da Unidade Básica de Saúde (UBS), o que eleva ainda mais seu custo de manutenção. A diferença entre o repasse do MS e o valor efetivamente gasto com a equipe é custeada pelo município, ficando a cargo do gestor a avaliação dos gastos em saúde. Mesmo considerando os dispositivos legais vigentes, que estabelecem regras para uso do dinheiro público, há grande autonomia do gestor na definição de valores e serviços a serem ofertados. Nesse processo, influenciado por diversos fatores que este estudo não conseguiu identificar, além da distância de grandes centros, também é possível a manifestação da influência do interesse político na contratação de profissionais com altos salários, mesmo em um contexto econômico desfavorável, sob a justificativa de que o investimento seria necessário para manter o profissional no município.

Essa ideia pode ser uma explicação nesse contexto de grande amplitude de valores salariais, considerando que as variáveis contextuais não exerceram influência sobre os salários pagos aos médicos contratados, e que importantes indicadores de desenvolvimento e desigualdade social, de saúde e de qualidade de assistência social, tais como IDH, Índice de Gini, IMRS e IVS não revelaram influência sobre o valor do salário pago a médicos contratados. E, mesmo em contextos muitos semelhantes, municípios vizinhos da Macro Norte pagam aos médicos contratados da APS valores muito discrepantes.

Faz-se necessário destacar que, com a 
adoção do Programa Previne Brasil ${ }^{33}$ pelo MS, que instituiu um novo modelo de financiamento federal para a APS a partir de janeiro de 2020, 37 municípios da Macro Norte (43\%) tiveram decréscimo dos valores a serem transferidos mensalmente para custeio da APS. Muitos dos quais são municípios de pequeno porte, pobres e com os mais baixos níveis de desenvolvimento humano da região ${ }^{34}$. Esse fato acentua ainda mais o subfinanciamento das equipes da $\mathrm{ESF}^{35}$, bem como a necessidade da racionalização e do planejamento do gasto em saúde com o objetivo de almejar melhores resultados assistenciais ${ }^{36}$. Aproximadamente $60 \%(n=48)$ dos 78 municípios da Macro Norte estudados possuem população de até dez mil habitantes, ao passo que $97 \%$ ( $n=76$ ) são classificados com IDH-M baixo ou médio. Ou seja, são municípios pequenos, com baixos níveis de desenvolvimento humano ${ }^{\mathbf{1 4}}$ e de pouca autonomia financeira ${ }^{37}$, sendo que quase a totalidade deles depende de transferências governamentais de recursos financeiros para realização das ações de saúde ${ }^{\mathbf{3 8}}$.

Em um contexto de crise política e econômica em que são adotadas medidas de austeridade fiscal com destaque para a Emenda Constitucional $n^{0} 95 / 2016$, que congela os gastos públicos em saúde por vinte anos, e com possibilidade real de contingenciamento de gastos para áreas prioritárias como saúde e educação, é imperativo que os gestores municipais adotem critérios claros para definição de salários para os médicos contratados para trabalhar na ESF. A criação de um teto salarial regional visando à redução da rotatividade e a avaliação técnica e criteriosa da necessidade de pessoal, bem como das formas de contratação e ingresso nas carreiras municipais, são caminhos a serem considerados.

Convém ressaltar que, no Brasil, a problemática do financiamento, da escassez e provisão de médicos é amplamente discutida na literatura científica ${ }^{\mathbf{2}, \mathbf{3}} \mathbf{5}$. Nesse contexto, a provisão de médicos para a APS encontra-se atualmente no centro de um crescente debate político, que inclui discussões sobre provimento emergencial de médicos para áreas desassistidas com mudanças na forma de alocação desses profissionais e formação de recursos humanos para o SUS que, junto ao financiamento, tem sido frequentemente apontada como uma estratégia essencial para a superação das iniquidades em saúde, das injustiças sociais e das desigualdades regionais no Brasil 15,38,39.

Estudos anteriores demonstraram que o PMMB foi preponderante na fixação de profissionais médicos nas localidades mais vulneráveis, longínquas e historicamente desprovidas desses profissionais e que trouxe expressivos resultados assistenciais nessas localidades, melhorando sobremaneira os indicadores assistenciais e de saúde $\mathbf{2}^{\mathbf{5}, \mathbf{4 0 - 4 2}}$. Considerando os resultados deste estudo, em que apenas a distância esteve associada aos salários dos médicos da ESF, recomenda-se a utilização do critério 'distância do município a centros regionais ou grandes centros' como parâmetro auxiliar para alocação de médicos nos programas governamentais de provimento de profissionais.

Os resultados deste estudo devem ser interpretados com cautela, dadas as limitações próprias do delineamento adotado. A principal limitação dos estudos ecológicos é a impossibilidade de relacionar exposição e desfecho no nível individual (falácia ecológica). Por sua vez, os estudos transversais descrevem apenas associações entre variáveis sem possibilidade de estabelecer causalidade. Também devem ser citadas as limitações inerentes às bases de dados secundários consultadas, que dependem da alimentação, completude e atualização garantidas pelos órgãos de origem. A variável dependente foi coletada pela SRS-Montes Claros junto a gestores municipais de saúde e não junto aos profissionais médicos.

Contudo, apesar de tais limitações, o tema pesquisado é muito relevante para a saúde pública e apresenta respostas úteis a gestores e pesquisadores. Trata-se do primeiro estudo realizado sobre a temática no norte de Minas Gerais. Espera-se que seus resultados 
contribuam para estimular as discussões sobre a necessidade da adoção de critérios para definição de salários para os médicos contratados para atuação na ESF, objetivando uma maior racionalização e transparência no uso do dinheiro público.

Ao mesmo tempo, é importante considerar as dificuldades enfrentadas nas diversas comunidades rurais da região, que refletem no interesse do profissional pelo trabalho no local, como, além da distância a grandes centros, a dificuldade de deslocamento devido à existência de barreiras geográficas, indisponibilidade de tecnologias como sinal de internet e de telefone, meios de transporte incompatíveis com a geografia ou relevo do terreno ou ausência de infraestrutura para hospedagem e alimentação, por exemplo.

\section{Conclusões}

Os resultados deste estudo evidenciam que não foram identificadas associações entre o salário e variáveis que caracterizam as condições sociodemográficas, geográficas e de assistência tais como IDH-M, Índice de Gini, Distância ao Centro Regional e Número de Médicos por
Mil Habitantes. Isso sugere que outros fatores, mais subjetivos e de difícil mensuração, como vontade política ou interesses particulares, podem estar envolvidos na determinação do valor dos salários pagos a médicos contratados da ESF. Portanto, percebe-se a necessidade de definição e implantação de parâmetros claros para contribuição de forma objetiva no estabelecimento do valor do salário de médicos contratados para a ESF.

\section{Colaboradores}

Pereira JA (0000-0003-0980-4454)*, Damasceno RF (0000-0002-9525-8527)*, Vieira MRM (0000-0001-5185-5381)* e Haikal DS (0000-0002-0331-0747)* contribuíram igualmente para a elaboração do manuscrito com as seguintes atividades: 1) concepção e o planejamento, análise e interpretação dos dados; 2) elaboração do rascunho e revisão crítica do conteúdo; e 3) aprovação da versão final do manuscrito. De Paula AMB (00000002-8715-0030)* contribui para a elaboração do manuscrito com a seguinte atividade: 1 ) participou da aprovação da versão final do manuscrito. 


\section{Referências}

1. Peruzzo HE, Bega AG, Lopes APAT, et al. The challenges of teamwork in the family health strategy. Esc. Anna Nery [internet]. 2018 ago [acesso em 2019 fev 2]; 22(4):e20170372. Disponível em: http:// www.scielo.br/scielo.php?script=sci_arttext\&pid $=$ S1414-81452018000400205.

2. Campos GVS, Pereira Júnior N. Primary care in Brazil, and the Mais Médicos (More Doctors) Program in the Unified Health System: achievements and limits. Ciênc. Saúde Colet. [internet]. 2016 set [acesso em 2018 dez 12]; 21(9):2655-2663. Disponível em: https://www.scielo.br/pdf/csc/v21n9/en_1413-8123csc-21-09-2655.pdf.

3. Stralen ACSV, Massote AW, Carvalho CL, et al. Percepção de médicos sobre fatores de atração e fixação em áreas remotas e desassistidas: rotas da escassez. Physis [internet]. $2017 \mathrm{jan} / \mathrm{mar}$ [acesso em 2018 dez 13]; 27(1):147-172. Disponível em: http://www. scielo.br/scielo.php?script=sci_arttext\&pid=S0103$-73312017000100147 \& \operatorname{lng}=$ en\&nrm=iso\&tlng=pt.

4. Poli Neto P, Faoro NT, Prado Júnior JC, et al. Variable compensation in Primary Healthcare: a report on the experience in Curitiba, Rio de Janeiro, Brazil, and Lisbon, Portugal. Ciênc. Saúde Colet. [internet]. 2016 [acesso em 2018 dez 18]; 21(5):1377-1388. Disponível em: http://www.scielo.br/scielo.php?script=sci arttext\&pid=S1413-81232016000501377\&lng=en\&nr $\mathrm{m}=$ iso\&tlng=en.

5. Kemper ES, Mendonça AVM, Sousa MF. The Mais Médicos (More Doctors) Program: panorama of the scientific output. Ciênc. Saúde Colet. [internet]. 2016 [acesso em 2018 dez 15]; 21(09):2785-2796. Disponível em: http://www.scielo.br/scielo.php?script=sci arttext\&pid $=$ S1413-81232016000902785\&lng=en\&n $\mathrm{rm}=\mathrm{iso} \&$ tlng $=\mathrm{en}$.

6. Cavalli LO, Rizzotto MLF, Guimarães ATB. Physicians in the external assessment process of the National Program for Access and Quality Improvement in Primary Care, cycles I and II - Brazil. Saúde debate [internet]. 2016 [acesso em $2018 \mathrm{dez} 19$ ]; 40(111):87-
100. Disponível em: http://www.scielo.br/scielo. php?pid=S0103-11042016000400087\&script=sci_ arttext\&tlng=en.

7. Scheffer M, coordenador. Demografia Médica no Brasil 2018 [internet]. São Paulo: FMUSP/CFM/CREMESP; 2018. [acesso em 2019 jun 23]. Disponível em: http://www.epsjv.fiocruz.br/sites/default/files/files/ DemografiaMedica2018\%20(3).pdf.

8. Cabreira FS, Ritter F, Aguiar VR, et al. Despesas municipais em atenção primária à saúde no Rio Grande do Sul, Brasil: um estudo ecológico. Cad. Saúde Pública [internet]. 2018 [acesso em 2020 maio 2]; 34(12):e00150117. Disponível em: http://cadernos.ensp.fiocruz.br/csp/public_site/arquivo/ CSP_1501_17_Despesa.pdf.

9. Tanaka OY, Tamaki EM. O papel da avaliação para a tomada de decisão na gestão de serviços de saúde. Ciênc. Saúde Colet. [internet]. 2012 [acesso em 2020 maio 3]; 17(4):821-828. Disponível em: https://www. scielo.br/pdf/csc/v17n4/v17n4a02.pdf.

10. Ney MS, Rodrigues PHA. Fatores críticos para a fixação do médico na Estratégia Saúde da Família. Physis [internet]. 2012 [acesso em 2018 nov 11]; 22(4):1293-1311. Disponível em: http://www.scielo.br/scielo.php?script=sci_arttext\&pid=S0103$-73312012000400003 \& \operatorname{lng}=e n \& n r m=$ iso\&tlng=pt.

11. Moreira LMC, Ferré F, Andrade EIG. Healthcare financing, decentralization and regional health planning: federal transfers and the healthcare networks in Minas Gerais, Brazil. Ciênc. Saúde Colet. [internet]. 2017 [acesso em 2019 jan 19]; 22(4):1245-1256. Disponível em: http://www.scielo.br/scielo.php?script=sci arttext\&pid=S1413-81232017002401245\&lng=en\&nr $\mathrm{m}=$ iso\&tlng=pt.

12. Alves MO, Magalhães SCM, Coelho BA. A regionalização da saúde e a assistência aos usuários com câncer de mama. Saúde Soc [internet]. 2017 [acesso em 2019 fev 25]; 26(1):141-154. Disponível em: http://www. scielo.br/scielo.php?script=sci_arttext\&pid=S0104- 
$-12902017000100141 \& \operatorname{lng}=$ en\&nrm=iso\&tlng=pt.

13. Damasceno RF, Caldeira AP. Teleconsultoria na atenção primária no norte de Minas Gerais: cenário e fatores associados à sua não utilização por médicos. Reciis [internet]. 2018 out/dez [acesso em 2019 jun 18]; 12(4):456-465. Disponível em: https://www. reciis.icict.fiocruz.br/index.php/reciis/article/ view/1312/2232.

14. Programa das Nações Unidas para o Desenvolvimento (PNUD). Atlas do Desenvolvimento Humano no Brasil [internet]. 2013. [acesso em 2018 ago 21]. Disponível em: http://atlasbrasil.org.br/2013/.

15. Menezes ASS, Alves MJS, Gomes TP, et al. Microcefalia relacionada ao vírus Zika e dinâmica familiar: perspectiva da mãe. Av Enferm [internet]. 2019 jan/ abr [acesso em 2019 jul 19]; 37(1):38-46. Disponível em: http://www.scielo.org.co/scielo.php?script=sci arttext\&pid=S0121-45002019000100038\&lng=en\&n $\mathrm{rm}=\mathrm{iso} \& \operatorname{tlng}=\mathrm{pt}$.

16. Leite ME, Melo MASS. Juventudes e espaço urbano: uma análise geográfica na cidade de

1. Montes Claros/MG. Caderno de Geografia [internet]. 2017 jan/mar [acesso em 2019 set 29]; 27(48):123-141. Disponível em: http://periodicos.pucminas.br/index.php/geografia/article/view/P.23182962.2017v27n48p123.

17. Rede Interagencial de Informações para a Saúde. Indicadores básicos para a saúde no Brasil: conceitos e aplicações [internet]. 2. ed. Brasília, DF: Opas; 2008 [acesso em 2017 dez 12]. Disponível em: http://www. ripsa.org.br/vhl/indicadores-e-dados-basicos-para-a-saude-no-brasil-idb/.

18. Ministério da Saúde. Datasus [internet]. [Brasília, DF]: Ministério da Saúde; [2017] [acesso em 2017 dez 12]. Disponível em: http://tabnet.datasus.gov.br/cgi/ deftohtm.exe?cnes/cnv/prid02MG.def.

19. Instituto Brasileiro de Geografia e Estatística. Censo 2010 [internet]. [Brasília, DF]: IBGE; [2017] [acesso em 2017 dez 12]. Disponível em: https://www.ibge. gov.br/estatisticas/downloads-estatisticas.html.

20. Field A. Descobrindo a Estatística Utilizando o SPSS. 2. ed. Porto Alegre: Artmed; 2009.

21. Martins PCR, Pontes ERJC, Paranhos Filho AC, et al. Gravidez na adolescência: estudo ecológico nas microrregiões de saúde do Estado do Mato Grosso do Sul, Brasil - 2008. Epidemiol. Serv. Saúde [internet]. 2014 [acesso em 2018 jun 22]; 23(1):91-100. Disponível em: http://www.scielo.br/scielo.php?script=sci arttext\&pid=S2237-96222014000100091\&lng=en\&n $\mathrm{rm}=\mathrm{iso} \&$ tlng $=\mathrm{pt}$.

22. Pereira AGL, Medronho RA, Escosteguy CC, et al. Spatial distribution and socioeconomic contexto of tuberculosis in Rio de Janeiro, Brazil. Rev. Saúde Pública [internet]. 2015 [acesso em 2019 mar 13]; 49:48. Disponível em: http://www.scielo.br/scielo.php?script=sci arttext\&pid=S0034-89102015000100234\&lng=en\&n $\mathrm{rm}=\mathrm{iso} \& \operatorname{tn} \mathrm{ln}=\mathrm{en} \& O R I G I N A L L A N G=e n$.

23. Oliveira BLCA, Luiz RR. Racial density and the socioeconomic, demographic and health context in Brazilian cities in 2000 and 2010. Rev. bras. epidemiol [internet]. 2019 [acesso em 2019 jul 3]; 22:E190036. Disponível em: https://www.ncbi.nlm.nih.gov/pubmed/31038617.

24. Clark J, Barnes A, Campbell M, et al. A life or "good death" situation? A worldwide ecological study of the national contexts of countries that have and have not implemented palliative care. Journal of Pain and Symptom Management [internet]. 2019 [acesso em 2019 jul 4]; 57(4):793-801. Disponível em: https:// www.ncbi.nlm.nih.gov/pubmed/30594568.

25. Keown P, McBride O, Twigg L, et al. Rates of voluntary and compulsory psychiatric in-patient treatment in England: an ecological study investigating associations with deprivation and demographics. Brit J Psychiat [internet]. 2016 [acesso em 2019 jul 15]; 209:157-161. Disponível em: https://www.ncbi.nlm. nih.gov/pubmed/27284079.

26. Costa SM, Lima CA, Nobre ALCSD, et al. Hypertension bearers with high risk/big risk of cardiovascu- 
lar diseases and socioeconomic and health indicators. Rev. Assoc. Med. Bras [internet]. 2018 jul [acesso em 2019 ago 1]; 64(7):601-610. Disponível em: http:// www.scielo.br/scielo.php?script=sci_arttext\&pid =S0104-42302018000700601.

27. Von Pressentin KB, Mash BJ, Esterhuizen TM. Examining the influence of family physician supply on district health system performance in South Africa: An ecological analysis of key health indicators. Afr J Prim Health Care Fam Med [internet]. 2017 [acesso em 2019 ago 7]; 9(1):el-e10. Disponível em: https:// www.ncbi.nlm.nih.gov/pubmed/28470076.

28. Martins Júnior DF, Felzemburg RDM, Dias AB, et al. Trends in mortality from ill-defined causes among the elderly in Brazil, 1979-2013: ecological study. Sao Paulo Med. J [internet]. 2016 [acesso em 2019 ago 11]; 134(5):437-45. Disponível em: https://www.ncbi.nlm. nih.gov/pubmed/27901244.

29. Galavote HS, Franco TB, Freitas PSS, et al. A gestão do trabalho na estratégia saúde da família: (des) potencialidades no cotidiano do trabalho em saúde. Saúde Soc [internet]. 2016 out/dez [acesso em 2019 ago 15]; 25(4):988-1002. Disponível em: http://www. scielo.br/scielo.php?script=sci_arttext\&pid=S0104$-12902016000400988 \& \operatorname{lng}=$ en\&nrm=iso\&tlng=pt.

30. Girardi SN, Carvalho CL, Wan Der Maas L, et al. Preferências para o trabalho na atenção primária por estudantes de medicina em Minas Gerais, Brasil: evidências de um experimento de preferência declarada. Cad. Saúde Pública [internet]. 2017 [acesso em 2019 set 3]; 33(8):e00075316. Disponível em: http://www. scielo.br/scielo.php?script=sci_arttext\&pid=S0102$-311 X 2017000805012 \& \operatorname{lng}=$ en\&nrm=iso\&tlng=pt.

31. Kotzee TJ, Couper ID. What interventions do South African qualified doctors think will retain them in rural hospitals of the Limpopo province of South Africa? Rural and Remote Health [internet]. 2006 [acesso em 2019 ago 14]; 6(3):e581. Disponível em: https://www.ncbi.nlm.nih.gov/pubmed/16965219.

32. Araújo CA, Michelotti FC, Ramos TKS. Government provision programs: profile and motivations of physi- cians who migrated from Provab to the More Doctors Program in 2016. Interface (Botucatu) [internet]. 2017 [acesso em 2019 ago 19]; 21(supl1):1217-1228. Disponível em: http://www.scielo.br/scielo.php?script=sci arttext\&pid=S1414-32832017000501217\&lng=en\&nr $\mathrm{m}=$ iso\&tlng=en.

33. Brasil. Ministério da Saúde. Portaria no 2.979, de 12 de novembro de 2019. Institui o Programa Previne Brasil, que estabelece novo modelo de financiamento de custeio da Atenção Primária à Saúde no âmbito do Sistema Único de Saúde, por meio da alteração da Portaria de Consolidação n ${ }^{0}$ 6/GM/MS, de 28 de setembro de 2017. Diário Oficial da União. 13 Nov 2019. [acesso em 2020 ago 6]. Disponível em: https://www. in.gov.br/en/web/dou/-/portaria-n-2.979-de-12-de-novembro-de-2019-227652180.

34. Brasil. Ministério da Saúde. Portaria n ${ }^{0} 173$, de 31 de janeiro de 2020. Dispõe sobre os municípios que apresentam decréscimo dos valores a serem transferidos, conforme as regras do financiamento de custeio da Atenção Primária à Saúde do Programa Previne Brasil. Diário Oficial da União. 31 Jan 2020. [acesso em 2020 ago 6]. Disponível em: https://www.in.gov. br/en/web/dou/-/portaria-n-173-de-31-de-janeiro-de-2020-240912938.

35. Tesser CD, Norman AH, Vidal TB. Acesso ao cuidado na Atenção Primária à Saúde brasileira: situação, problemas e estratégias de superação. Saúde debate [internet]. 2018 [acesso em 2020 maio 5]; 42(esp1):361378. Disponível em: https://www.scielo.br/pdf/sdeb/ v42nspel/0103-1104-sdeb-42-spe01-0361.pdf.

36. Cecilio LCO, Reis AAC. Notes on persistent challenges for basic health care in Brazil. Cad. Saúde Pública [internet]. 2018 [acesso em 2019 ago 25]; 34(8):e00056917. Disponível em: https://www.ncbi. nlm.nih.gov/pubmed/30133653.

37. Caetano CCR, Ávila LAC, Tavares M. A relação entre as transferências governamentais, a arrecadação tributária própria e o índice de educação dos municípios do estado de Minas Gerais. Revista de Administração Pública [internet]. 2017 [acesso em 2019 ago 22]; 51(5):897-916. Disponível em: http://www. 
scielo.br/scielo.php?script=sci_arttext\&pid=S0034$-76122017000500897 \& \operatorname{lng}=$ en\&nrm=iso\&tlng=pt.

38. Massardi WO, Abrantes LA. Dependência dos municípios de Minas Gerais em relação ao FPM. Revista de Gestão, Finanças e Contabilidade [internet]. 2016 [acesso em 2019 ago 23]; 6(1):173-187. Disponível em: https://www.revistas.uneb.br/index.php/financ/article/view/1127/1465.

39. Medina MG, Almeida PF, Lima JG, et al. Programa Mais Médicos: mapeamento e análise da produção acadêmica no período 2013-2016 no Brasil. Saúde debate [internet]. 2018 [acesso em 2020 maio 5]; 42(esp1):346-360. Disponível em: https://www. scielo.br/pdf/sdeb/v42nspel/0103-1104-sdeb-42spe01-0346.pdf.

40. Telles H, Silva ALA, Bastos C. Programa Mais Médicos do Brasil: a centralidade da relação médico-usuário para a satisfação com o programa. Cad. CRH [internet]. 2019 [acesso em 2019 set 12]; 32(85):101-123. Disponível em: http://www.scielo.br/scielo.php?script=sci_
arttext\&pid=S0103-49792019000100101\&lng=en\&n $\mathrm{rm}=\mathrm{iso} \& \operatorname{lng}=\mathrm{pt}$.

41. Castro MC, Massuda A, Almeida G, et al. Brazil's unified health system: the first 30 years and prospects for the future. The Lancet [internet]. 2019 [acesso em 2019 set 18]; 394(10195):345-356. Disponível em: https://www.thelancet.com/action/showPdf?p ii $=$ S0140-6736\%2819\%2931243-7.

42. Facchini LA, Tomasi E, Dilélio AS. Qualidade da Atenção Primária à Saúde no Brasil: avanços, desafios e perspectivas. Saúde debate [internet]. 2018 [acesso em 2020 maio 5]; 42(esp1):208-223. Disponível em: https://www.scielo.br/pdf/sdeb/v42nspel/0103-1104sdeb-42-spe01-0208.pdf.

Recebido em 01/10/2019

Aprovado em 07/05/2020

Conflito de interesses: inexistente

Suporte financeiro: não houve 